\title{
Stokes Flow around Rotating Axially Symmetric Pervious Body
}

\author{
D. K. Srivastava \\ Department of Mathematics, B.S.N.V. Post Graduate College \\ (Lucknow University, Lucknow), Lucknow(Uttar Pradesh)-226001, India \\ dksflow@hotmail.com
}

(Received January 5, 2012; accepted April 19, 2012)

\begin{abstract}
In this paper, the problem of slowly rotating pervious axially symmetric body with source at its centre placed in an incompressible viscous fluid has been tackled. The method of separation of variables has been used and the general solution in terms of Legendre functions and Whittaker's polynomial is given. As a first approximation, for $n=1$, the results are in confirmation with spherical body. It is found that the effect of source at the centre is to reduce the resulting moment. Further, it has been conjectured that the results of couple for other bodies ( i.e., for $\mathrm{n} \geq 2$ ) can also be obtained on the same ground but presently it is beyond the scope of the paper and would likely to appear in the future paper.
\end{abstract}

Keywords: Slow rotation, Axially symmetric bodies, Source, Viscous fluid, Stokes approximation.

\section{NOMENCLATURE}

$\mathrm{e}_{\mathrm{x}}$
$\mathrm{e}_{\mathrm{r}}$
$\mathrm{e}_{\theta}$
$\mathrm{e}_{\varphi}$
$\mathrm{M}$
$\mathrm{M}(\mathrm{a}, \mathrm{b}, \mathrm{z})$
$\mathrm{n}$
$\mathrm{p}$
$\mathrm{Q}$

unit vector along $\mathrm{x}$-axis unit vector along radial direction unit vector along transverse direction unit vector along azimuthal direction moment

Kummer function positive integer pressure strength of fluid source

$\begin{array}{ll}\mathrm{r}, \theta, \varphi & \text { spherical polar coordinates } \\ \mathrm{s}=\mathrm{Q} / \mathrm{va} & \text { source parameter } \\ \mathrm{u} & \text { velocity vector } \\ \mathrm{W}_{\mathrm{k}, \mathrm{t}} & \text { Whittakar's function } \\ \rho & \text { fluid density } \\ \sigma_{\mathrm{r} \varphi} & \text { viscous stress } \\ \mathrm{v} & \text { kinematic viscosity } \\ \Omega & \text { angular velocity } \\ \nabla^{2} & \text { Laplacian operator }\end{array}$

\section{INTRODUCTION}

The problem of determining the couple experienced by an axially symmetric body, rotating steadily in a viscous and incompressible fluid has engaged the attention of many authors in the amongst others by Collins (1955), Childress (1964), Davis (1980), Datta (1973), Datta and Srivastava (2000), Dennis et. al. (1981), Hackborn (1990), Happel and Brenner (1965), Jeffery (1915), Lamb (1952), Kanwal (1961), Macaraeg (1986), Ranger (1971), Rieutord and Valdettaro (1997), Smith (1981), Tanzosh and Stone (1994), Vasudevaiah and Majhi (1982), Datta and Srivastava (2000), Kim and Choi (2002), Felderhof (2007), Murthy et al. (2007), Marcello (2008), Wan (2009), Arbaret et al. (2011), Ashmawy (2011), Chang and Keh (2012).

Slow rotation of spheroids(including the disc) in an infinite fluid was first solved by Jeffrey (1915) using curvilinear coordinates. His approach was later extended to the spherical lens, torus, and other axisymmetric shapes. Collins (1955) have drawn an analogy between the slow steady rotation of a solid of revolution about its axis in a viscous fluid and the motion, with constant velocity. The flow generated by an axi-symmetric body due to the rotation about its axis of symmetry in a viscous fluid was studied by R.P. Kanwal (1961) by treating the flow to be Stokesian(i.e. by neglecting non linear convective terms) and found a general formula for couple as a limit on the toroidal velocity. Rubinow and Keller (1961) have considered the force on a spinning sphere which is moving through an incompressible viscous fluid by employing the method of matched asymptotic expansions to describe the asymmetric flow. Brenner (1961) also obtained some general results for the drag and couple on an obstacle which is moving through the fluid. Childress (1964) has investigated the motion of a sphere moving through a rotating fluid and calculated a correction to 
the drag coefficient. Thomas and Walter (1964) considered the rotation of a sphere in a visco-elastic fluid $B^{\prime}$ (known as Walters fluid). The solution is obtained for the velocity using regular perturbation method with rotation Reynolds number as the perturbation parameter. Manohar (1965) considered the flow of slightly visco elastic fluid due to slow rotation of a sphere contained in a cylinder by neglecting nonlinear terms. Wakiya (1967) numerically evaluated the drag and angular velocity experienced by freely rotating spheres and compared with calculated from corresponding approximate formulae known before. Barrett (1967) has tackled the problem of impulsively started sphere rotating with angular velocity $\Omega$ about a diameter. He modified the standard time-dependent boundary layer equation to give series solutions satisfying all the boundary conditions and gave solutions that are applicable at small times for non-zero Reynolds numbers. He found that the velocity components decay algebraically rather than exponentially at large distances. Rao et al. (1969) have tackled the problem of slow steady rotation of a sphere in a micropolar fluid. Kanwal (1970) has considered a disk performing simple harmonic rotary oscillations about its axis of symmetry in a non-conducting viscous fluid which is at rest at infinity. Ranger (1971) tackled the problem of axially symmetric flow past a rotating sphere due to a uniform stream of infinity. He has shown that leading terms for the flow consists of a linear superposition of a primary Stokes flow past a non-rotating sphere together with an anti symmetric secondary flow in the azimuthal plane induced by the spinning sphere. Cooley (1971) has investigated the problem of fluid motion generated by a sphere rotating close to a fixed sphere about a diameter perpendicular to the line of centers in the case when the motion is sufficiently slow to permit the linearization of the Navier-Stokes equations by neglecting the inertia terms. He used a method of matched asymptotic expansions to find asymptotic expressions for the forces and couples acting on the spheres as the minimum clearance between them tends to zero. In his paper, the forces and couples are shown to have the form $\mathrm{a}_{0} \ln \varepsilon+\mathrm{a}_{1}+\mathrm{o}(\varepsilon \ln$ $\varepsilon$ ), where $\varepsilon$ is the ratio of the minimum clearance between the spheres and the radius of the rotating sphere and where $a_{0}$ and $a_{1}$ are found explicitly. Using toroidal coordinates, an exact solution is derived for the velocity field induced in two immiscible semi-infinite fluids possessing a plane interface, by the slow rotation of an axially symmetric body partly immersed in each fluid by Schneider et al. (1973). Takagi (1974) has considered the flow around a spinning sphere moving in a viscous fluid. Takagi (1974) has studied the Stokes flow for the case in which two solid spheres in contact are steadily rotating with different angular velocities about their line of centers. Takagi (1977) further studied the problem of steady flow which is induced by the slow rotation of a solid sphere immersed in an infinite incompressible viscous fluid, on the basis of NavierStokes equations. He obtained the solution in the form of power series with respect to Reynolds number. Ramkissoon (1977) considered steady rotation of an axi-symmetric body in a micropolar fluid by neglecting non-linear terms. A fundamental singular solution due to a point force was derived and the method of associated matrices is used as an alternative method. He derived a general formula for couple similar to that of Kanwal (1961). Gierszewski and Cheffy (1978) studied the problem of rotation of an isolated triaxial ellipsoid suspended in slow viscous flow. Drew (1978) has found the force on a small sphere translating relative to a slow viscous flow to order of the $1 / 2$ power of Re for two different fluid flows far from the sphere, namely pure rotation and pure shear. For pure rotation, the correction of this order to the Stokes drag consists of an increase in the drag. Kim (1980) has calculated the torque and frictional force exerted by a viscous fluid on a sphere rotating on the axis of a circular cone of arbitrary vertex angle about an axis perpendicular to the cone axis in the Stokes approximation. Fosdick and Kao (1980) considered the slow steady rotation of a sphere in a simple fluid of order 4 in order to account the formation of a cap observed experimentally near the pole of the rotating sphere in a non- Newtonian fluid. Smith (1981) studied the influence of rotation in slow viscous flows. Dennis et al. (1981) considered the slow steady rotation of a sphere in a viscous fluid. The couple was obtained for a wide range of Reynolds number using finite difference like special technique. Rao and Iyengar $(1981,1983)$ studied the flow due to the slow rotation and rotary oscillations of a spheroid in a micropolar fluid neglecting the convective terms. Davis and Brenner (1986) have used the matched asymptotic expansion methods to solve the problem of steady rotation of a tethered sphere at small, non-zero Reynolds numbers. They obtained first order Taylor number correction to both the Stokes-law drag and Kirchhoff's law couple on the sphere for Rossby numbers of order unity. O'Neill and Yano (1988) derived the boundary condition at the surfactant and substrate fluids caused by the slow rotation of a solid sphere which is partially submerged in the substrate fluid. The problems of slow rotation and rotary oscillations of an approximate sphere in a micropolar fluid was studied by Iyengar and Srinivasacharya (1995, 2001). Ranger(1996) has found an exact solution of the Navier-Stokes equations for the axi-symmetric motion(with swirl) representing exponentially timedependent decay of a solid sphere translating and rotating in a viscous fluid relative to a uniform stream whose speed also decays exponentially with time. $\mathrm{He}$ also described a similar solution for the twodimensional analogue where the sphere is replaced by a circular cylinder of infinite length. Tekasakul et al. (1998) have studied the problem of the rotatory oscillation of an axi-symmetric body in an axisymmetric viscous flow at low Reynolds numbers. They evaluated numerically the local stresses and torques on a selection of free, oscillating, axi-symmetric bodies in the continuum regime in an axi-symmetric viscous incompressible flow. Datta and Srivastava (2000) have tackled the problem of slow rotation of a sphere with fluid source at its centre in a viscous fluid. In their investigation, it was found that the effect of fluid source at the centre is to reduce the couple on slowly rotating sphere about its diameter. Datta and Pandya (2001) tackled the problem of axial symmetric rotation of a partially immersed body in a liquid with a surfactant layer. Kim and Choi (2002) conducted the numerical simulations for laminar flow past a sphere rotating in the streamwise direction, in order to investigate the effect of the rotation on the 
characteristics of flow over the sphere. Tekasakul and Loyalka (2003) have investigated the rotary oscillations of several axisymmetric bodies in axisymmetric viscous flows with slip. A numerical method based on the Green's function technique is used and analytic solutions for local stress and torque on spheres and spheroids as function of the frequency parameter and the slip coefficients are obtained. They have analyzed that in all cases, slip reduces stress and torque, and increasingly so with the increasing frequency parameter. Davis (2006) obtained the expression for force and torque on a rotating sphere close to and within a fluid-filled rotating sphere. Felderhof (2007) considered the Stokes problem of impulsively twisted sphere in an incompressible viscous fluid. Murthy et al. (2007) have discussed the flow generated by the slow steady rotation of a permeable sphere about its axis of symmetry in an incompressible micropolar fluid. Marcello (2008) has introduced new exact analytic solutions for the rotational motion of a axially symmetric rigid body having two equal principal moments of inertia and subjected to an external torque which is constant in magnitude. Felderhof (2011) studied the transient flow caused by a sudden impulse or twist applied to a sphere immersed in a viscous incompressible fluid. Arbaret et al. (2011) discussed the effect of shape and orientation on rigid particle rotation and matrix deformation in simple shear flow. Ashmawy (2011) discussed the rotational motion of an arbitrary axisymmetric body in a viscous fluid using a combined analytical-numerical technique. Mendez (2011) discussed the problem of a flow model for the settling velocities of non spherical particles in creeping motion.

The purpose of this paper is to study slow rotation of a axisymmetric body, assumed to be pervious, with source at its centre. Let ' $Q$ ' be the source strength and $\Omega$ be the angular velocity of the slowly rotating axially symmetric body. We assume that the source strength ' $Q$ ' is of the same order as the angular velocity $\Omega$ of the rotating body so that non-linear inertia terms could be neglected. In this situation the total flow consists of only the source solution superimposed on the Stokes solution and the Stokes drag and couple are not affected by the source. On the other hand when we assume that ' $\mathrm{Q}$ ' is large enough so that $\mathrm{Q} \Omega$ is not negligible, the inertia terms being non-linear, cannot be altogether omitted, the equation, however, can still be linearized by assuming that the velocity perturbation due to the source flow on account of the Stokes flow is small so that the terms containing square of angular velocity can be neglected. This assumption is justifiable at least in the vicinity of the axi-symmetric body where the Stokes approximation is valid too. The present problem corresponds to the problem of Stokes flow past a sphere with source at its centre investigated by Datta (1973) and slow rotation of sphere with source at its centre by Datta and Srivastava (2000). The results of which have found application in investigating the diffusiophoresis target efficiency for evaporation or condensing drop by Placek and Peters (1980).

\section{Formulation Of The Problem}

Let us consider a pervious axisymmetric body with source of strength $\mathrm{Q}$ at its centre generating radial flow field around it in an infinite expanse of incompressible fluid of density $\rho$ and kinematic viscosity $v$. The body also rotates with small steady angular velocity $\Omega$ so that terms of order $\mathrm{O}\left(\Omega^{2}\right)$ may be neglected but the terms of order $\mathrm{O}(\mathrm{Q} \Omega)$ retained.

The motion is governed by Navier-Stokes equations

$\mathbf{u} \cdot \operatorname{grad} \mathbf{u}=-(1 / \rho) \operatorname{grad} p+\nu \nabla^{2} \mathbf{u}$,

and continuity equation

$\operatorname{div} \mathbf{u}=0$,

together with no-slip boundary condition

$\mathbf{u}=\Omega \hat{\mathbf{e}}_{\mathrm{x}} \times \mathrm{r} \hat{\mathbf{e}}_{\mathrm{r}}$, on the surface, $\mathrm{r}=\mathrm{r}(\theta)$,

where ' $a$ ' is axial length, and the condition of vanishing of velocity at far off points

$\mathbf{u}=0$, at infinity as $\mathrm{r} \rightarrow \infty$.

It will be convenient to work in spherical polar coordinates $(\mathrm{r}, \theta, \varphi)$ with $\mathrm{x}$-axis as the polar axis. We non-dimensionalize the space variables by ' $a$ ' (axial length), velocity by $a \Omega$ and pressure by $\rho v \Omega$. The velocity vector, in non-dimensional form, may be express as

$\mathbf{u}=\frac{\mathrm{Q}}{\mathrm{a}^{2} \mathrm{r}^{2}} \hat{\mathbf{e}}_{\mathrm{r}}+\mathrm{a} \Omega\left(\mathrm{u}_{\mathrm{r}} \hat{\mathbf{e}}_{\mathrm{r}}+\mathrm{u}_{\theta} \hat{\mathbf{e}}_{\theta}\right)+\mathrm{a} \Omega \mathrm{u}_{\varphi} \hat{\mathbf{e}}_{\varphi}$

and pressure(non-dimensional form) as

$\mathrm{p}=\rho \vee \Omega\left[p_{0}+p_{1}(r, \theta)\right]$

where $\mathrm{u}_{\mathrm{r}}, \mathrm{u}_{\theta}, \mathrm{u}_{\varphi}$ are velocity components in spherical polar coordinates with unit vectors $\hat{\mathbf{e}}_{\mathrm{r}}, \hat{\mathbf{e}}_{\theta}, \hat{\mathbf{e}}_{\varphi}$.

On substituting the values of $\mathbf{u}$ and $\mathrm{p}$ from Eq. (5) and Eq. (6) in Eq. (1), we get

$$
\begin{aligned}
& \nabla^{2} \mathrm{u}_{\mathrm{r}}-\frac{2 \mathrm{u}_{\mathrm{r}}}{\mathrm{r}^{2}}-\frac{2}{\mathrm{r}^{2} \sin \theta} \frac{\partial}{\partial \theta}\left(\mathrm{u}_{\theta} \sin \theta\right) \\
& =\frac{\partial}{\partial \mathrm{r}}\left[\mathrm{p}_{0}+\frac{\mathrm{Q}^{2}}{\rho \mu \Omega \mathrm{a}^{4} \mathrm{r}^{4}}+\mathrm{p}_{1}+\frac{\mathrm{su}_{\mathrm{r}}}{\mathrm{r}^{2}}\right] \\
& \nabla^{2} \mathrm{u}_{\theta}+\frac{2}{\mathrm{r}^{2}} \frac{\partial \mathrm{u}_{\mathrm{r}}}{\partial \theta}-\frac{\mathrm{u}_{\theta}}{\mathrm{r}^{2} \sin ^{2} \theta} \\
& -\frac{\mathrm{s}}{\mathrm{r}^{3}}\left[\frac{\partial\left(\mathrm{ru}_{\theta}\right)}{\partial \mathrm{r}}-\frac{\partial \mathrm{u}_{\mathrm{r}}}{\partial \theta}\right]=\frac{1}{\mathrm{r}} \frac{\partial}{\partial \theta}\left(\mathrm{p}_{1}+\frac{\mathrm{su}_{\mathrm{r}}}{\mathrm{r}}\right) \\
& \nabla^{2} \mathrm{u}_{\varphi}-\frac{\mathrm{u}_{\varphi}}{\mathrm{r}^{2} \sin ^{2} \theta}=\frac{\mathrm{s}}{\mathrm{r}^{3}} \frac{\partial}{\partial \mathrm{r}}\left(\mathrm{ru}_{\varphi}\right)
\end{aligned}
$$

where $\mathrm{s}=\mathrm{Q} / \mathrm{va}$ is source parameter and $\nabla^{2}$ is the Laplacian operator in spherical polar coordinates defined as 
D. K. Srivastava / JAFM, Vol. 6, No. 3, pp. 435-442 2013.

$$
\nabla^{2} \equiv \frac{1}{r^{2}}\left[\frac{\partial}{\partial r}\left(r^{2} \frac{\partial}{\partial r}\right)+\frac{1}{\sin \theta} \frac{\partial}{\partial \theta}\left(\sin \theta \frac{\partial}{\partial \theta}\right)+\frac{1}{\sin ^{2} \theta} \frac{\partial^{2}}{\partial \varphi^{2}}\right]
$$

On assuming the polar axis, in spherical polar coordinates, in the direction of angular velocity $\Omega$, we have $\mathrm{u}_{\mathrm{r}}=\mathrm{u}_{\theta}=0$. The Eq. (7a) and Eq. (7b) provides

$$
\mathrm{p}_{0}=-\frac{\mathrm{Q}^{2}}{2 v \Omega \mathrm{a}{ }^{4} \mathrm{r}^{4}}+\mathrm{k}(\theta) \text { and } \mathrm{p}_{1}=\mathrm{p}_{1}(\mathrm{r})
$$

where $k(\theta)$ is constant function of integration. So the expression of pressure will be

$$
\mathrm{p}=\rho v \Omega\left[-\frac{\mathrm{Q}^{2}}{2 v \Omega \mathrm{a}^{4} \mathrm{r}^{4}}-\mathrm{p}_{1}(\mathrm{r})\right]+\mathrm{k}(\theta)
$$

Now Eq. (7c) is to be solved under the boundary conditions

$$
\left\{\begin{array}{l}
\mathrm{u}_{\varphi}=\mathrm{a} \Omega \sin \theta \text { at } \quad \mathrm{r}=\mathrm{r}(\theta) \\
\mathrm{u}_{\varphi} \rightarrow 0 \quad \text { as } \quad \mathrm{r} \rightarrow \infty
\end{array}\right.
$$

\section{SOLUTION}

Let us take the solution of Eq. (7c) in the form

$\mathrm{u}_{\varphi}=\mathrm{F}(\mathrm{r}, \mathrm{s}) \cdot \mathrm{G}(\theta)$

After evaluating the constituent parts, Eq. (7c) reduces to

$$
\frac{\mathrm{FG}}{\mathrm{r}^{2}}\left[\begin{array}{l}
\frac{1}{\mathrm{~F}} \frac{\mathrm{d}}{\mathrm{dr}}\left(\mathrm{r}^{2} \frac{\mathrm{dF}}{\mathrm{dr}}\right)-\frac{\mathrm{s}}{\mathrm{r}} \frac{1}{\mathrm{~F}} \frac{\mathrm{d}}{\mathrm{dr}}(\mathrm{rF}) \\
+\frac{1}{\mathrm{G}} \frac{\mathrm{d}}{\mathrm{d} \mu}\left\{\left(1-\mu^{2}\right) \frac{\mathrm{dG}}{\mathrm{d} \mu}\right\}-\frac{1}{1-\mu^{2}}
\end{array}\right]=0
$$

where $\mu=\cos \theta$, on writing,

$$
\frac{1}{\mathrm{~F}}\left[\frac{\mathrm{d}}{\mathrm{dr}}\left(\mathrm{r}^{2} \frac{\mathrm{dF}}{\mathrm{dr}}\right)-\frac{\mathrm{s}}{\mathrm{r}} \frac{\mathrm{d}}{\mathrm{dr}}(\mathrm{rF})\right]=\mathrm{n}(\mathrm{n}+1)
$$

Or

$$
\frac{\mathrm{d}}{\mathrm{dr}}\left(\mathrm{r}^{2} \frac{\mathrm{dF}}{\mathrm{dr}}\right)-\frac{\mathrm{s}}{\mathrm{r}} \frac{\mathrm{d}}{\mathrm{dr}}(\mathrm{rF})-\mathrm{n}(\mathrm{n}+1) \mathrm{F}=0
$$

with ' $n$ ' as positive integer. In particular, for $n=1$, we get the equation, solution of which contribute in slowly rotating sphere with source at its centre. Viz.; the equation will be

$$
\frac{\mathrm{d}}{\mathrm{dr}}\left(\mathrm{r}^{2} \frac{\mathrm{dF}}{\mathrm{dr}}\right)-\frac{\mathrm{s}}{\mathrm{r} d \mathrm{~d}}(\mathrm{rF})-2 \mathrm{~F}=0
$$

Now, with the help of Eq. (14), Eq. (13) reduces to

$$
\begin{aligned}
& \frac{d}{d \mu}\left\{\left(1-\mu^{2}\right) \frac{d G}{d \mu}\right\}+\left\{n(n+1)-\frac{1}{1-\mu^{2}}\right\} G=0, \\
& \mu=\cos \theta
\end{aligned}
$$

in this case too, for $\mathrm{n}=1$, we can have

$\frac{\mathrm{d}}{\mathrm{d} \mu}\left\{\left(1-\mu^{2}\right) \frac{\mathrm{dG}}{\mathrm{d} \mu}\right\}+\left\{2-\frac{1}{1-\mu^{2}}\right\} \mathrm{G}=0$,

which has obvious solution, $\mathrm{G}(\theta)=\sin \theta$.

In general, the solution of Eq. (16) can easily be expressed in terms of Legendre Functions (Abramowitz and Stegun, 1968)

$$
\begin{aligned}
\mathrm{G}(\theta) & =\mathrm{G}_{\mathrm{n}}(\theta) \\
& =\mathrm{P}_{\mathrm{n}}^{1}(\mu), \mu=\cos \theta \\
& =-\sqrt{1-\mu^{2}} \mathrm{P}_{\mathrm{n}}^{\prime}(\mu) \\
& =-\frac{\mathrm{d}}{\mathrm{d} \theta}\left\{\mathrm{P}_{\mathrm{n}}(\cos \theta)\right\},
\end{aligned}
$$

which, for $n=1$, provide $G(\theta)=\sin \theta$.

Now, we discuss the general solution of Eq. (14), we take, $\mathrm{s} / \mathrm{r}=\mathrm{z}$, then Eq. (14) reduces to

$\frac{\mathrm{d}^{2} \mathrm{~F}}{\mathrm{dz}^{2}}+\frac{\mathrm{dF}}{\mathrm{dz}}-\left\{\frac{1}{\mathrm{z}}+\frac{\mathrm{n}(\mathrm{n}+1)}{\mathrm{z}^{2}}\right\} \mathrm{F}=0$

which is second order linear differential equation in standard form with $\mathrm{Q}(\mathrm{z})=-\left\{\frac{1}{\mathrm{z}}+\frac{\mathrm{n}(\mathrm{n}+1)}{\mathrm{z}^{2}}\right\}$, $\mathrm{P}(\mathrm{z})=1$, Converting it into normal form, with $\mathrm{F}(\mathrm{z})=$ $\mathrm{u}(\mathrm{z}) \mathrm{e}^{-\mathrm{z} / 2}$, this equation further reduces to

$\frac{\mathrm{d}^{2} \mathrm{u}}{\mathrm{dz}^{2}}+\left\{-\frac{1}{4}-\frac{1}{\mathrm{z}}-\frac{\mathrm{n}(\mathrm{n}+1)}{\mathrm{z}^{2}}\right\} \mathrm{u}(\mathrm{z})=0$

which is a Whittaker's equation (Abramowitz and Stegun, 1968) for

$\mathrm{k}=-1$ and $\frac{1}{4}-\mathrm{t}^{2}=-\mathrm{n}(\mathrm{n}+1)$

in particular, for $\mathrm{n}=1, \mathrm{k}=-1, \mathrm{t}= \pm 3 / 2, \mathrm{n}=2, \mathrm{k}=-1$, $\mathrm{t}$ $= \pm 5 / 2, \mathrm{n}=3, \mathrm{k}=-1, \mathrm{t}= \pm 7 / 2$ and so on.

And in general, $\mathrm{t}^{2}=\frac{1}{4}+\mathrm{n}(\mathrm{n}+1)$.

Now, the solution of Whittaker's equation will be in terms of Whittaker's function (Abramowitz and Stegun, 1968) defined as

$\mathrm{W}_{\mathrm{k}, \mathrm{t}}(\mathrm{z})=\mathrm{e}^{-\frac{\mathrm{z}}{2}} \mathrm{z}^{\frac{1}{2}+\mathrm{t}} \mathrm{U}\left(\frac{1}{2}+\mathrm{t}-\mathrm{k}, 1+2 \mathrm{t}, \mathrm{z}\right)$

where 
D. K. Srivastava / JAFM, Vol. 6, No. 3, pp. 435-442 2013.

$$
\mathrm{U}(\mathrm{a}, \mathrm{b}, \mathrm{z})=\frac{\pi}{\sin \pi \mathrm{b}}\left[\begin{array}{l}
\frac{\mathrm{M}(\mathrm{a}, \mathrm{b}, \mathrm{z})}{(2+a-b) !(b+1) !} \\
-\left\{\mathrm{z}^{(1-b)}\right\} \frac{\mathrm{M}(1+\mathrm{a}-\mathrm{b}, 2-\mathrm{b}, \mathrm{z})}{(1+a) !(3-b) !}
\end{array}\right]
$$

with $\mathrm{a}, \mathrm{b}$ are real numbers and $\mathrm{M}(\mathrm{a}, \mathrm{b}, \mathrm{z})$ is Kummer function (Abramowitz and Stegun, 1968) defined as

$$
\begin{aligned}
\mathrm{M}(\mathrm{a}, \mathrm{b}, \mathrm{z})=1 & +\frac{\mathrm{a}}{\mathrm{b}} \mathrm{z}+\frac{(\mathrm{a})_{2}}{(\mathrm{~b})_{2}} \frac{\mathrm{z}^{2}}{2 !}+\frac{(\mathrm{a})_{3}}{(\mathrm{~b})_{3}} \frac{\mathrm{z}^{3}}{3 !} \\
& +\ldots \ldots \ldots+\frac{(\mathrm{a})_{n}}{(\mathrm{~b})_{n}} \frac{\mathrm{z}^{\mathrm{n}}}{\mathrm{n} !}+\ldots \ldots .
\end{aligned}
$$

with $(a)_{n}=a(a+1) \ldots . .(a+n-1),(a)_{0}=1$. The restrictions on this Whittaker's functions are $-\pi<\arg \mathrm{z}<\pi$, $\mathrm{k}=\frac{\mathrm{b}}{2}-2, \mathrm{t}=\frac{\mathrm{b}}{2}-\frac{1}{2}$.
So the solution of Eq. (14) may be expressed in terms of Whittaker's function as

$$
\begin{aligned}
& \mathrm{F}(\mathrm{r}, \mathrm{s})=\mathrm{e}^{-\frac{\mathrm{s}}{2 \mathrm{r}}} \mathrm{W}_{\mathrm{k}, \mathrm{t}}\left(\frac{\mathrm{s}}{\mathrm{r}}\right), \mathrm{k}=-1, \\
& \mathrm{t}= \pm \sqrt{\frac{1}{4}+\mathrm{n}(\mathrm{n}+1)}
\end{aligned}
$$

Therefore, the azimuthal component, of velocity can be expressed as

$\mathrm{u}_{\varphi}=\mathrm{e}^{-\frac{\mathrm{s}}{2 \mathrm{r}}} \mathrm{W}_{\mathrm{k}, \mathrm{t}}\left(\frac{\mathrm{s}}{\mathrm{r}}\right) \cdot \mathrm{G}_{\mathrm{n}}(\theta)$

Now, we use this result to verify the solution obtained by authors (Datta and Srivastava, 2000) for the rotation of sphere with source at its centre.

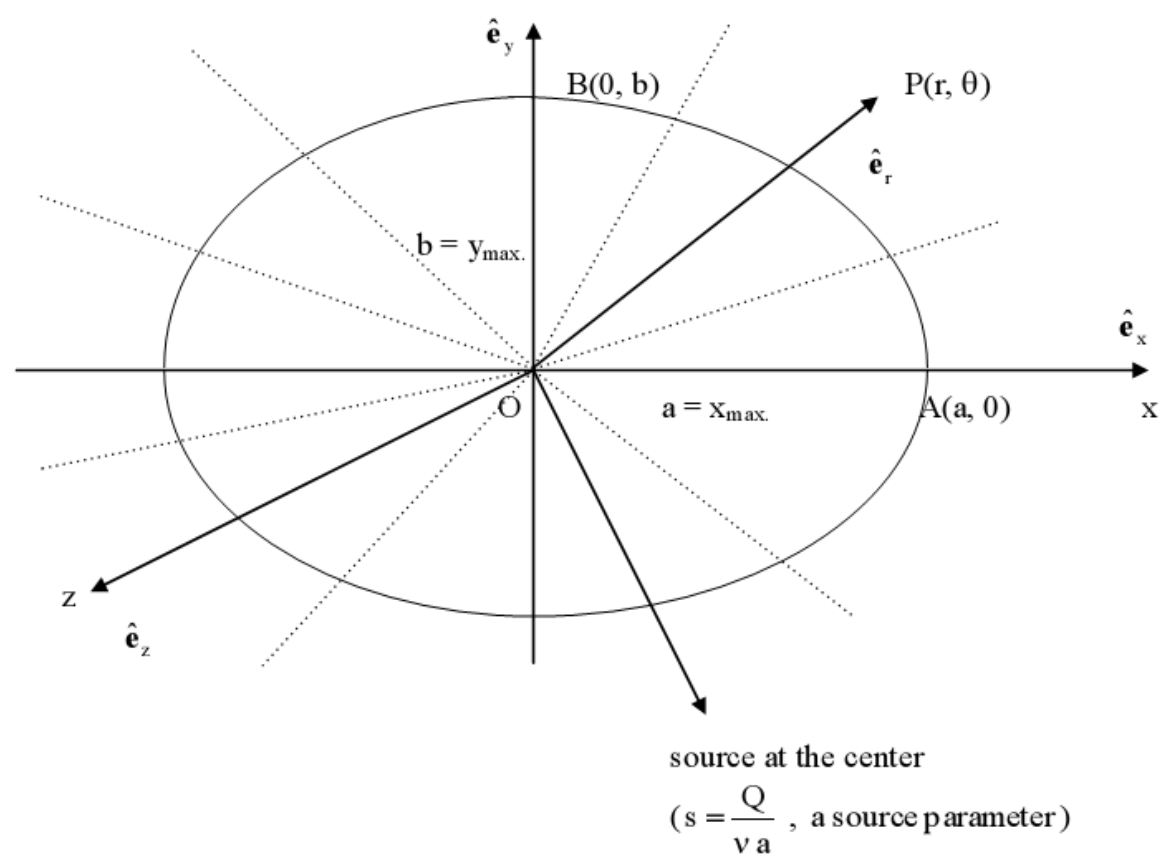

Fig. 1. Rotating axially symmetric pervious body having angular velocity $\Omega$ with source at its center

\section{Rotation OF THE SPHERE}

\subsection{The case with $n=1$}

With some manipulations, it can be easily analyzed that the azimuthal component of velocity in the case of sphere rotating with small angular velocity $\Omega$, under the specific boundary conditions on the surface of sphere, $r=a$,

$\mathbf{u}=\mathrm{a} \Omega \hat{\mathbf{e}}_{\mathrm{x}} \times \hat{\mathbf{e}}_{\mathrm{r}}$,

and $\mathbf{u}=0$, at infinity as $\mathrm{r} \rightarrow \infty$

comes out to be

$u_{\varphi}=r \sin \theta\left[\frac{s^{2}}{r^{2}}-2 \frac{s}{r}+2-2 e^{-\frac{s}{r}}\right]\left[s^{2}-2 s+2-2 e^{-s}\right]^{-1}$

Then, the couple on the sphere required to maintain the motion is obtained by integration of viscous stress

$$
\begin{aligned}
& \sigma_{\mathrm{r} \varphi}=\mu \Omega\left[\frac{\partial \mathrm{u}_{\varphi}}{\partial \mathrm{r}}-\frac{\mathrm{u}_{\varphi}}{\mathrm{r}}\right]_{\mathrm{r}=1} \\
& =2 \mu \Omega \sin \theta\left[-\mathrm{s}^{2}+\mathrm{s}\left(1-\mathrm{e}^{-\mathrm{s}}\right)\right]\left[\mathrm{s}^{2}-2 \mathrm{~s}+2-2 \mathrm{e}^{-\mathrm{s}}\right]^{-1}
\end{aligned}
$$

thus, the moment of the required couple is given by 
D. K. Srivastava / JAFM, Vol. 6, No. 3, pp. 435-442 2013.

$$
\begin{aligned}
& \mathrm{M}=-\int_{\theta=0}^{\pi} \quad \sigma_{\mathrm{r} \varphi} \mathrm{a}^{3} 2 \pi \sin ^{3} \theta \mathrm{d} \theta \\
& \frac{16}{3} \pi \mathrm{a}^{3} \mu \Omega\left[\mathrm{s}\left(1-\mathrm{e}^{-\mathrm{s}}\right)-\mathrm{s}^{2}\right]\left[2-2 \mathrm{~s}+\mathrm{s}^{2}-2 \mathrm{e}^{-\mathrm{s}}\right]^{-1}
\end{aligned}
$$

which match with result of moment given by authors (Datta and Srivastava, 2000).

\section{NUMERICAL DISCUSSION}

When we take up the case of small 's' (source parameter), we get from Eq. (4) and Eq. (5)

$$
\mathrm{M}=8 \pi \mathrm{a}^{3} \mu \Omega\left[1-\frac{\mathrm{s}}{12}\right]
$$

which provides the classical value $\mathrm{M}_{0}=8 \pi \mathrm{a}^{3} \mu \Omega$ for $\mathrm{s}=0$.
Also, for large values of 's', we have the approximation as

$\mathrm{M}=\frac{16}{3} \pi \mathrm{a}^{3} \mu \Omega\left[1+\frac{1}{\mathrm{~s}}\right]$

We find that $\mathrm{M} \rightarrow \frac{2}{3} \mathrm{M}_{0}$, as $\mathrm{s} \rightarrow \infty$.

The variation of moment coefficient $C_{M}=\frac{M}{M_{0}}$ with source parameter $\mathrm{s}=\frac{\mathrm{Q}}{\mathrm{va}}$, where $\mathrm{M}_{0}$ is the moment for $\mathrm{s}$ $=0$, has been shown in Fig. 2, which clearly shows that the effect of source at the centre of the sphere is to reduce the moment ultimately to two third of its value in the absence of source.

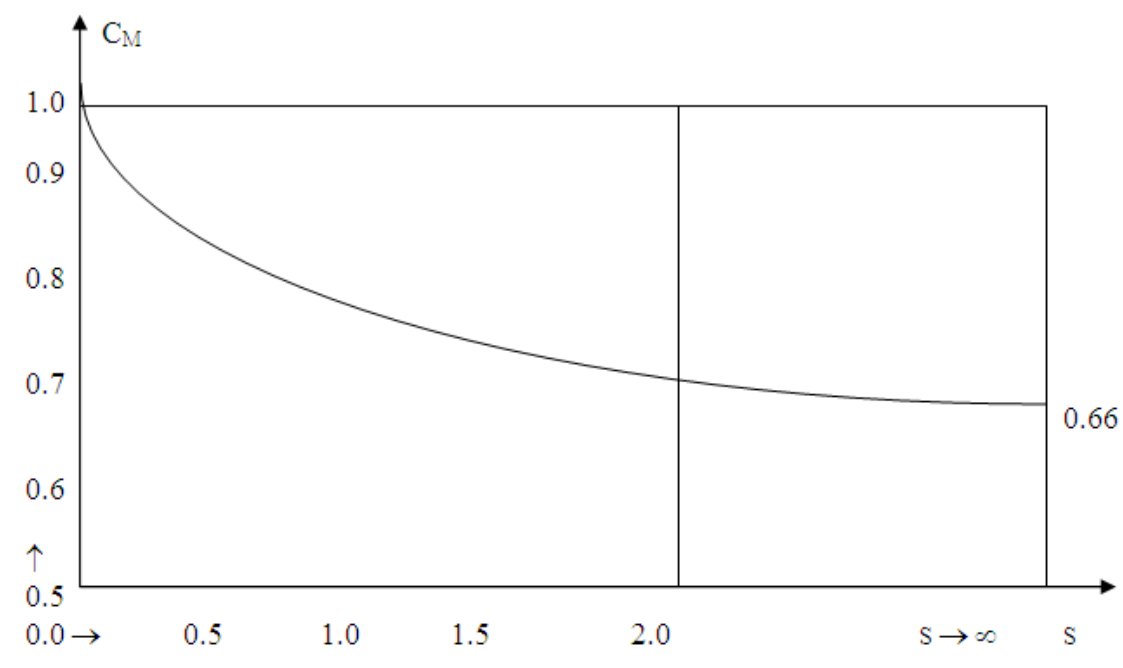

Fig. 2. Variation of moment coefficient $C_{M}$ with source parameter 's'

\section{CONCLUSION}

The sections 4 and 5 of the paper are devoted to the verification of the general solution for azimuthal component of velocity in case of spherical body and subsequently other results such as viscous stress and moment have been discussed for the same body. Further, at this stage, we may only conjecture, that for $n>1(n \geq 2)$, there are some other axi-symmetric bodies, which could be of spheroidal shape, slightly deformed sphere etc. We reserve this part of the work for future investigation. Based on section 5, we conjectured that the effect of fluid source at the centre of axially symmetric slowly rotating body is to reduce the moment.

\section{ACKNOWLEDGEMENTS}

Author is very much thankful to University Grants Commission, New Delhi, India for providing financial support under the major research project[39$55 / 2010(\mathrm{SR})]$ scheme awarded at the department of mathematics, B.S.N.V. P.G. College(University of Lucknow), Lucknow(U.P.)-226001, India. I am also thankful to the authorities of the college for providing me basic infrastructure facilities throughout the preparation of the manuscript.

\section{REFERENCES}

Abramowitz, M. and I. A. Stegun (1968). A hand book of mathematical functions. Dover publications.

Arbaret, L.; Mancktelow, Neil S. and Burg, Jean-Pierre (2011). Effect of shape and orientation on rigid particle rotation and matrix deformation in simple shear flow, Journal of Structural Geology, 23(1), 113-125.

Ashmawy, E. A. (2011). Slip at the surface of a general axi-symmetric body rotating in a viscous fluid, Archives of Mechanics, 63(4), 341-361.

Barret, K. E. (1967). On the impulsively started rotating sphere, Journal of Fluid Mechanics, 27, 779-788. 
Brenner, H. (1961). The slow motion of a sphere through a viscous fluid towards a plane surface, Chemical Engineering Science, 16, 242-251.

Chang, Y.C. and Keh, Huan J. (2012). Creeping Flow rotation of a slip spheroid about its axis of revolution, Theoretical and Computational Fluid Dynamics, 26(1-4), 173-183.

Childress, S. (1964). The slow motion of a sphere in a rotating viscous fluid. Journal of Fluid Mechanics. $20,305-314$.

Collins, W. D. (1955). On the steady rotation of a sphere in a viscous fluid, Mathematika, 2(1), 42-47.

Cooley, M. D. A. (1971). The slow rotation in a viscous fluid of a sphere close to another fixed sphere about a diameter perpendicular to the line of centres, The Quarterly Journal of Mechanics and Applied Mathematics, 24, 237-250.

Datta, S. (1973). Stokes flow past a sphere with a source at its centre. Math. Vesnik. Vol. 10, pp. 227 229

Datta, S. and D. K. Srivastava (2000, Feb). Slow rotation of sphere with a source at its centre in a viscous flow. Proc. (Math. Sci.), Ind. Acad. Sciences., 110(1), 1-4.

Datta, S. and N. Pandya (2001). Axial symmetric rotation of a partially immersed body in a liquid with a surfactant layer, Sadhana, 26(4), 353-362.

Davis, A. M. J. (1980). The torque on a rotating body in a liquid with a surfactant layer and its relation to the virtual mass of a heaving body. The Quarterly Journal of Mechanics and Applied Mathematics, $33,337-355$

Davis, A. M. J. and H. Branner (1986). Steady motion of a tethered sphere at small, non-zero Reynolds and Taylor numbers, Journal of Fluid Mechanics, $168,151-167$.

Davis, A. (2006, Nov.). Force and torque on a rotating sphere close to and within a fluid-filled rotating sphere, Bull. Amer. Phys. Soc., $59^{\text {th }}$ Annual Meeting of the APS Division of Fluid Dynamics, Tompa Bay, Florida.

Dennis S. C. R.; Singh, S. N. and Ingham, D.B. (1980). The steady flow due to a rotating sphere. Journal of Fluid Mechanics, 101, 257-279.

Drew, D. A. (1978). The force on a small sphere in slow viscous flow, Journal of Fluid Mechanics, 88, 393-400.

Felderhof, B. U. (2007a). Flow caused by a square force pulse applied to a sphere immersed in a viscous incompressible fluid, Physics of Fluids, 19, 093102.
Felderhof, B. U. (2007b). Transient flow caused by a sudden impulse or twist applied to a sphere immersed in a viscous incompressible fluid, Physics of Fluids, 19, 073102.

Felderhof, B. U. (2007c). Effect of fluid compressibility on the flow caused by sudden impulse applied to a sphere immersed in a viscous fluid, Physics of Fluids, 19, 126101.

Fosdick, R. L. and B. G. Kao (1980). Steady flow of a simple fluid around a rotating sphere, Rheologica Acta, 19(6), 675-697.

Gierszewski, P. J. and C. E. Chaffey (1978). Rotation of an isolated triaxial ellipsoid suspended in slow viscous flow, Canadian Journal of Physics, 56(1), 6-11.

Hackborn, W. W. (1990). Asymmetric Stokes flow between parallel planes due to a rotlet. Journal of Fluid Mechanics. 218, 257-279.

Happel, J. and H. Brenner (1965). Low Reynolds number hydrodynamic. Academic press.

Iyengar, T. K. V. and D. Srinivasa Charya (1995). Slow steady rotation of an approximate sphere in an incompressible micropolar fluid, International Journal of Engineering Science, 33, 867-877.

Jeffery, G. B. (1915). Steady rotation of a solid of revolution in a viscous fluid. Proc. Lond. Math. Soc., 14, 327-338.

Kanwal, R. P. (1961). Slow steady rotation of axially symmetric bodies in a viscous fluid, Journal of Fluid Mechanics, 10, 17-24.

Kanwal, R. P.(1970). note on slow rotation or rotary oscillations of axi-symmetric bodies in hydrodynamics and magneto hydrodynamics. Journal of Fluid Mechanics, 41(4), 721-726.

Kim, M. U. (1980). Slow viscous rotation of a sphere on the axis of a circular cone, Physics of Fluids, 23(6), 11268-1269.

Kim, D. J. and H. C. Choi (2002). Laminar flow past a sphere rotating in the streamwise direction. Journal of Fluid Mechanics, 461, 365-386.

Lamb, H. (1954). Hydrodynamics. Dover publications.

Macaraeg, M. G. (1986). A mixed pseudo spectral/ finite difference method for the axi-symmetric flow in a heated, rotating spherical shell, Journal of Computational Physics. 62(2), 297-320.

Manohar, G. Palekar (1965). Slow rotation of a sphere in a slightly visco elastic fluid contained in a infinite cylinder, Zeitschrift für angewandte Mathematik und Physik ZAMP, 16, 502-515. 
D. K. Srivastava / JAFM, Vol. 6, No. 3, pp. 435-442 2013.

Mendez, Y. (2011) A flow model for the settling velocities of non spherical particles in creeping motion, Journal of Applied Fluid Mechanics, 4(4), 65-75.

Murthy, J. V. Ramana; Iyengar, N. Ch. S.N. and Aparna, P. (2007). Slow steady rotation of a permeable sphere in an incompressible micropolar fluid, IeJEMTA(Engineering and Mathematics), 2, 44-60.

O’Neill, M. E. and H. Yano (1988). The slow rotation of a sphere straddling a free surface with a surfactant layer, The Quarterly Journal of Mechanics and Applied Mathematics, 41(4), 479501.

Placek, T. D. and L. K. Peters (1980). A hydrodynamic approach to particle target efficiency in the presence of diffusiophoresis. Journal of Aerosol Science, 11, 521-533.

Ramkissoon, H. (1977). Slow steady rotation of an axially symmetric body in a micropolar fluid, Applied Scientific Research, 33(3-4), 243-257.

Ranger, K. B. (1971). Slow viscous flow past a rotating sphere. Proc. Camb. Phil. Soc., 69, 333-336.

Ranger, K. B. (1996a). An exact solution of Reynolds viscous flow equations for the time dependent motion of a sphere in an infinite liquid, La - Societe - Royle - du - Canada - L - Academie - des Sciences - Comptes - Rendus Mathematiques(Mathematical report), 18(4), 143148.

Ranger, K. B. (1996b). Time-dependent decay of the motion of a sphere translating and rotating in a viscous liquid, The Quarterly Journal of Mechanics and Applied Mathematics,49(4), 621-633.

Rao, S. K. Lakshmana; Ramacharyulu, N.Ch.P. and Rao, P. Bhujanga (1969). Slow steady rotation of a sphere in a micropolar fluid, International Journal of Engineering Science, 7, 905-916.

Rao, S. K. Lakshmana and T. K. V. Iyengar, (1981). Slow steady rotation of a spheroid in an incompressible micro polar fluid, International Journal of Engineering Science, 19, 655-687.

Rao, S. K. Lakshmana and T. K. V. Iyengar, (1983). Rotary oscillations of a spheroid in an incompressible micro polar fluid, International Journal of Engineering Science, 21, 973-987.

Riutord, M. and L. Valdettaro, (1997). Inertial waves in rotating spherical shell. Journal of Fluid Mechanics, 341, 77-99.
Romano, M. (2008). Exact analytic solutions for the rotation of an axially symmetric rigid body subjected to a constant torque, Celestial Mechanics and Dynamical Astronomy, 101(4), 375-390.

Rubinow, S. I. and Kellar, J. B. (1961). The transverse force on a spinning sphere moving in a viscous fluid, Journal of Fluid Mechanics, 11(3), 447-459.

Schneider, J. C.; O’Neill, M. E. and Brenner, Howard (1973). On the slow viscous rotation of a body straddling the interface between two immiscible semi-infinite fluids, Mathematika, 20, 175-196.

Smith, S. H. (1995). A note on Stokes flow due to a line rotlet. Mathematika. 42(1), 127-132.

Takagi, H. (1974a). On the slow motion of a sphere in a viscous fluid, Journal of the Physical Society of Japan, 37(2), 505-510.

Takagi, H.i (1974b). The Stokes flow past an impulsively started circular cylinder, Journal of the Physical Society of Japan, 37(2), 558-560.

Takagi, H. (1977). Viscous flow induced by slow rotation of a sphere, Journal of the Physical Society of Japan, 42(1), 319-325.

Tanzosh, J. P. and H. A. Stone, (1994). Motion of a rigid in a rotating viscous flow, an integral equation approach. Journal of Fluid Mechanics, 275, 225256.

Tekasakul, P. and S. K. Loyalka, (2003). Rotatory oscillatory of axi-symmetric viscous flow with slip: Numerical solutions for spheres and spheroids, International Journal for Numerical Methods in Fluids, 41(8), 823-940.

Tekasekul, P.; Tompson, R. V. and Loyalka, S. K. (1998). Rotary oscillations of arbitrary axisymmetry bodies in an axi-symmetric viscous flow: numerical solutions, Physics of Fluids, 10(11), 2797-2818.

Thomas, R. H. and K. Walters, (1964). The motion of an elastic-viscous liquid due to a sphere rotating about its diameter, Q.J.M.A.M., Vol. 17, pp. 39-54.

Vasudevaiah, M. and Majhi, S. N. (1982). Matched solutions of slow viscous flow past a rotating sphere. J. Math. Phys. Sc., 16(1), 43-55.

Wakiya, S. (1967). Slow motion of a viscous fluid around two spheres, Journal of the Physical Society of Japan, 22(4), 1101-1109.

Wan, Y. W. (2009). Slow rotation of an axially symmetric slip particle about its axis of revolution, CMES, Vol. 53, pp. 73-53. 